\title{
Decaying two-dimensional turbulence undergoes statistical heating
}

\author{
J. G. Esler* \\ Department of Mathematics, University College London, London, England \\ R. K. Scott \\ School of Mathematics and Statistics, University of St. Andrews, St. Andrews, Scotland
}

(Received 8 September 2019; accepted 30 March 2020; published 2 July 2020)

\begin{abstract}
An emergent property of decaying two-dimensional turbulence is shown to be a weak but persistent statistical heating, or tendency towards clustering of like-signed vortices. The rate of this heating, which is driven by changes to the vortex population due to vortex mergers and straining events, provides a strong constraint on both vortex decay laws and kinetic theories of vortex interactions. A quasi-equilibrium statistical theory determines the energy spectrum from just the vortex interaction energy and bulk information about the number density of the vortices. The emergent heating rate is shown to determine the upscale transfer of energy, and is a powerful constraint on power-law descriptions of the evolving vortex population.
\end{abstract}

DOI: 10.1103/PhysRevFluids.5.074601

\section{INTRODUCTION}

The basic phenomenology of decaying two-dimensional classical turbulence (2DCT) is well documented [1-5]. Starting from random initial conditions, a population of vortices becomes established and evolves, through like-signed mergers and straining out of vortices ("vortex thinning" [6]), to become ever larger in size and fewer in number. The focus of this work will be the related question of how exactly the statistical configuration, or temperature in the sense found in Ref. [7], of the vortex population evolves as this process unfolds. Is the trend towards like-signed clusters of vortices or opposite-signed dipoles? What processes determine the evolution?

Arguably the simplest measure [8] of the evolution of the configuration is the normalized vortex interaction energy $\varepsilon$ [9]. In both the point vortex system and its finite core extensions, $\varepsilon$ is defined to be the difference between the actual fluid energy of a distribution of vortices, and the expected energy were those same vortices randomly distributed in the domain, normalized by the standard deviation of the same random rearrangement process. Strongly positive $\varepsilon$ is associated with like-signed clustering ("negative temperature") and strongly negative $\varepsilon$ with dipole formation ("positive temperature"). The statistical heating rate will be defined here to be $\dot{\varepsilon}$, i.e., the rate at which the vortex population is evolving towards a clustered state (if the system is heating $\dot{\varepsilon}>0$ ) or a dipole state (if the system is cooling, $\dot{\varepsilon}<0$ ). (Note that the apparent contradiction of statistical heating leading to negative temperatures is resolved if one recalls that negative temperature states are "hotter" than positive ones, and arise when the the addition of energy to a closed system with a bounded phase-space causes the statistical temperature [10] to pass from $+\infty$ to $-\infty$.)

In two-dimensional quantum turbulence (2DQT), in which there is no analog of merger events and opposite-signed vortex dipoles annihilate when their separation becomes small, it is established that $\dot{\varepsilon}>0$ because of "evaporative heating" [11] due to the annihilations. Evaporative heating can be

\footnotetext{
*j.g.esler@ucl.ac.uk
} 
understood using "punctuated equilibrium" point vortex models [12,13], which capture the essential dynamics of 2DQT in the limit where the vortex core (or healing length) is small compared to the vortex separation. In the conservative dynamics between dipole annihilation events, energy conservation requires that the formation of a dipole must be compensated by a tendency towards like-signed clustering elsewhere in the domain. If the dipole is subsequently annihilated then the remaining, relatively more clustered, vortices will necessarily have higher $\varepsilon$.

In contrast to 2DQT, most simple models of decaying 2DCT, e.g., the authors of Refs. [12-14], characterized the evolution of the 2DCT vortex field purely in terms of mergers of like-signed vortices. It is important to note that the numerical results found in Ref. [6], showing that vortex thinning (an approximate analog of the vortex annihilation of 2DQT) can dominate the 2DCT spectral energy transfer in forced-dissipative simulations, does raise a question mark over this merger-only approach. Nevertheless, if mergers alone are considered, the same punctuated equilibrium argument given above leads to the conclusion that $\dot{\varepsilon}<0$, i.e., mergers lead to cooling. However, theoretical arguments based on maximizing mixing entropy [15,16], identify the end state of 2DCT with negative temperature states $(\varepsilon \gg 0)$, which imply heating has taken place. If the vortex merger paradigm found in Refs. [12-14] is accepted, these results appear to be in contradiction, and a key aim of this work is to resolve this apparent paradox.

In Sec. II below, a set of ensembles of high-resolution simulations of 2DCT are analyzed to measure the emergent heating rate. This heating rate is then shown to provide a powerful constraint on (i) the evolution of the energy spectrum in 2DCT, (ii) the coefficients in power-law descriptions of the evolving vortex populations, and (iii) the nature of any kinematic model of mergers and thinning in 2DCT. In Se. III the implications are discussed and conclusions are drawn.

\section{METHOD AND RESULTS}

\section{A. 2DCT simulations}

To simulate high-Reynolds number 2DCT, the regularized 2D Euler equations,

$$
\left(\partial_{t}+\boldsymbol{u} \cdot \nabla\right) \omega=D, \quad \boldsymbol{u}=\nabla^{\perp}\left(\Delta^{-1} \omega\right)
$$

where $\omega$ is the vorticity, are solved on a $2 \pi \times 2 \pi$ doubly periodic domain $\mathcal{D}$, using the CLAM algorithm [17] on a base grid of $512^{2}$ (by some measures equivalent to using a pseudospectral scheme with grid $8192^{2}$ ). The regularizing dissipation $D$ used by CLAM is the systematic removal of vortex filaments by contour surgery once they reach scales of approximately $1 / 16$ of the base grid-scale, thereby removing high-wave-number enstrophy from the system. Three ensembles of ten simulations, which differ in their initial conditions, are executed. The initial conditions $\omega(\boldsymbol{x}, 0)$ consist of a random excitation with a top-hat energy spectrum, in a band of wave numbers centered on $k_{i}=16,32$, and 64 , respectively.

To demonstrate the insensitivity of the main results to the choice of numerical scheme, the $k_{i}=32$ ensemble is repeated using a pseudospectral scheme on a $2048^{2}$ computational grid, with three different choices for the dissipation term $D$. The first is hyperdiffusion $D=-\mu_{h} \Delta^{4} \omega$ with $\mu_{h}=10 \cdot 2^{-5} \omega_{\text {rms }} k_{\max }^{8}$, the second is a time-varying diffusion $D=v_{v}(t) \Delta \omega$ with $v_{v}(t)=$ $2 \cdot 2^{-5} \omega_{\mathrm{rms}} k_{\max }^{2}\|\omega\|_{\infty}(t) /\|\omega\|_{\infty}(0)$, and the third is a standard constant (Navier-Stokes) diffusion $D=v_{c} \Delta \omega$ with $v_{c}=5 \cdot 2^{-5} \omega_{\mathrm{rms}} k_{\max }^{2}$. Here $\omega_{\mathrm{rms}}$ is the initial root mean squared vorticity and $k_{\max }$ is the maximum resolved wave number. The schemes differ in the amount of energy lost during the simulations. In the spin-up period, in which vortices are formed from the random initial conditions $\left(0<t / t_{\text {eddy }}<15\right.$ where $\left.t_{\text {eddy }}=4 \pi / \omega_{\text {rms }}\right)$, the energy loss is (CLAM, hyperdiffusion, time-varying diffusion, constant diffusion $)=(3.20,0.68,2.45,4.60) \%$, and for the main part of the simulations used for the results below $\left(15<t / t_{\text {eddy }}<280\right)$ it is $(0.86,0.02,3.14,6.84) \%$. The significantly higher-energy loss rate of the constant diffusion simulations might be be expected to influence the results below, which has motivated consideration of the range of schemes presented. 


\section{B. Ghost vortex dynamics}

A key tool used to analyze the 2DCT simulations above will be the following finite core extension of the point vortex model, here named ghost vortex dynamics (GVD), which describes the motion of $N$ axisymmetric vortices with invariant vorticity profiles $\omega_{i}(r)(i=1, \ldots, N)$. The evolution of the vortex centroids $\boldsymbol{x}_{i}=\left(x_{i}, y_{i}\right)^{T}$ is governed by Hamilton's equations

$$
\Gamma_{i} \frac{d x_{i}}{d t}=-\frac{\partial H}{\partial y_{i}}, \quad \Gamma_{i} \frac{d y_{i}}{d t}=\frac{\partial H}{\partial x_{i}}
$$

where

$$
H\left(x_{1}, \ldots, x_{N}\right)=-\frac{1}{2} \sum_{i=1}^{N} \sum_{j=1}^{N} G^{i j}\left(x_{i}, x_{j}\right)
$$

and $\Gamma_{i}=2 \pi \int_{0}^{\infty} \omega_{i}(r) r d r$ are the vortex circulations. The two-body interaction terms in $H$ are

$$
G^{i j}\left(\boldsymbol{x}_{i}, \boldsymbol{x}_{j}\right)=\int_{\mathcal{D}^{2}} \omega_{i}\left(\left|\boldsymbol{x}-\boldsymbol{x}_{i}\right|\right) G\left(\boldsymbol{x}, \boldsymbol{x}^{\prime}\right) \omega_{j}\left(\left|\boldsymbol{x}^{\prime}-\boldsymbol{x}_{j}\right|\right) d \boldsymbol{x} d \boldsymbol{x}^{\prime}
$$

with $G\left(\boldsymbol{x}, \boldsymbol{x}^{\prime}\right)$ the usual Green's function for the Laplacian in $\mathcal{D}$. Point vortex dynamics is recovered in a singular limit [18].

\section{Measurement methodology for $\varepsilon$}

To measure $\varepsilon$ in the 2DCT simulations, at each instant of time all coherent vortices in $\mathcal{D}$ are first identified using the algorithm found in Ref. [4]. All vortices thus identified are then mapped to the unique circular Gaussian vortex with the same centroid, circulation and self-enstrophy [19]. These vortices are then considered in the GVD model, and the exact GVD definition of $\varepsilon$

$$
\varepsilon=\frac{H-\mu_{H}}{\sigma_{H}},
$$

is used to calculate $\varepsilon$. Here $\mu_{H}=\langle H\rangle_{0}$ and $\sigma_{H}=\left\langle\left(H-\mu_{H}\right)^{2}\right\rangle_{0}^{1 / 2}$ are the mean and standard deviation of $H$ over the uniform ensemble $\langle\cdot\rangle_{0}$, in which all vortex centroids are randomly positioned in $\mathcal{D}$. A straightforward calculation (see Appendix 1) gives

$$
\mu_{H}=\sum_{k}^{\prime} \frac{1}{k^{2} \Pi(k)} \quad \text { and } \quad \sigma_{H}^{2}=\sum_{k}^{\prime} \frac{1}{k^{4} \Pi(k)^{2}}
$$

where the sum is over all independent integer wave numbers, $k=|\boldsymbol{k}|$, and $\Pi(k)^{-1}=\sum_{i=1}^{N} \hat{\omega}_{i}(k)^{2}$ with $\hat{\omega}_{i}(k)=\int_{0}^{\infty} \omega_{i}(r) \mathbf{J}_{0}(k r) r d r$ the Hankel transform of $\omega_{i}(r)$.

Notice that the GVD Hamiltonian $H$ is equal to the fluid energy obtained from the filtered vorticity field $\omega_{g}(\boldsymbol{x}, t)=\sum_{i=1}^{N} \omega_{i}\left(\left|\boldsymbol{x}-\boldsymbol{x}_{i}(t)\right|\right)$ generated by the vortex identification algorithm described above. Evidently $H$ is not equal to the exact 2DCT fluid energy obtained from $\omega$, but fluctuates randomly according to the goodness of fit of $\omega_{g}$ to $\omega$, without a significant trend $H=(0.903 \pm 0.034) E$ (standard deviation given).

\section{Main results: Emergent heating rate}

Figure 1 shows the evolution of $\varepsilon(t)$ across the three 2DCT simulation ensembles. A persistent heating is observed in each ensemble member over the full duration of the simulations, during which the number of vortices decreases by over an order of magnitude. To understand what is driving the heating, first note that, as discussed above, the total fluid energy $E$ in 2DCT is conserved to an excellent approximation. To a reasonable approximation, the GVD Hamiltonian $H \approx 0.9 E$ can also be considered to be conserved. Consequently, changes in $\varepsilon$ must be driven primarily by changes in $\mu_{H}$ and $\sigma_{H}$ due to $\Pi(k)$ changing as the vortex population evolves. The evolving population can be 

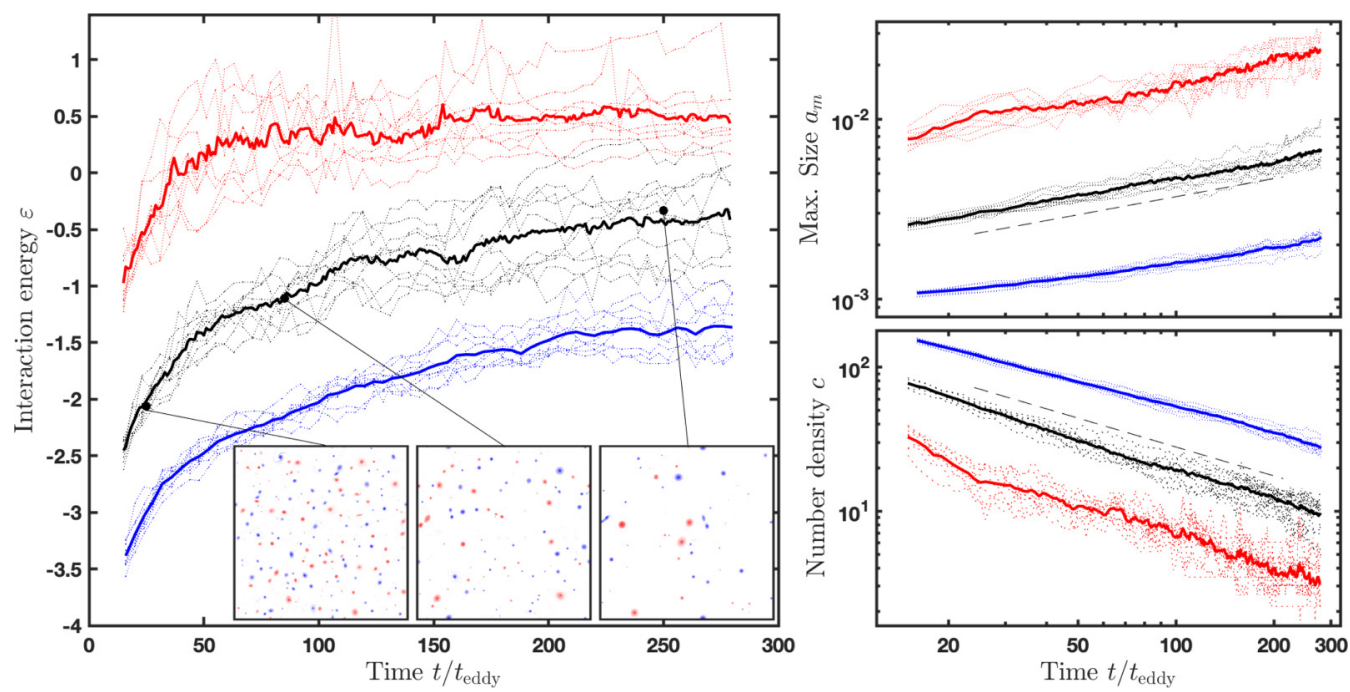

FIG. 1. Left: Time evolution of the vortex interaction energy $\varepsilon(t)$ in simulations (solid lines: ensemble average; light curves: individual runs) with narrowband initial conditions centered on $k_{i}=16$ (red), $k_{i}=32$ (black), and $k_{i}=64$ (blue). Insets: Snapshots of vorticity. Upper right: Evolution of fit to max. Vortex size $a_{m}(t)$ in the three ensembles (dashed line, $1 / 3$ power law). Lower right: Vortex number density $c(t)$ (dashed line, $-2 / 3$ power law).

largely described by a measure of the maximum vortex area $a_{m}(t)$ and the vortex population density $c(t)$, plotted in the right panels, see discussion below for details.

Figure 2 shows the ensemble average evolution of $\varepsilon(t)$ in the $k_{i}=32$ simulations when different numerical schemes are used. It is clear that the heating effect is robust and quantitatively almost

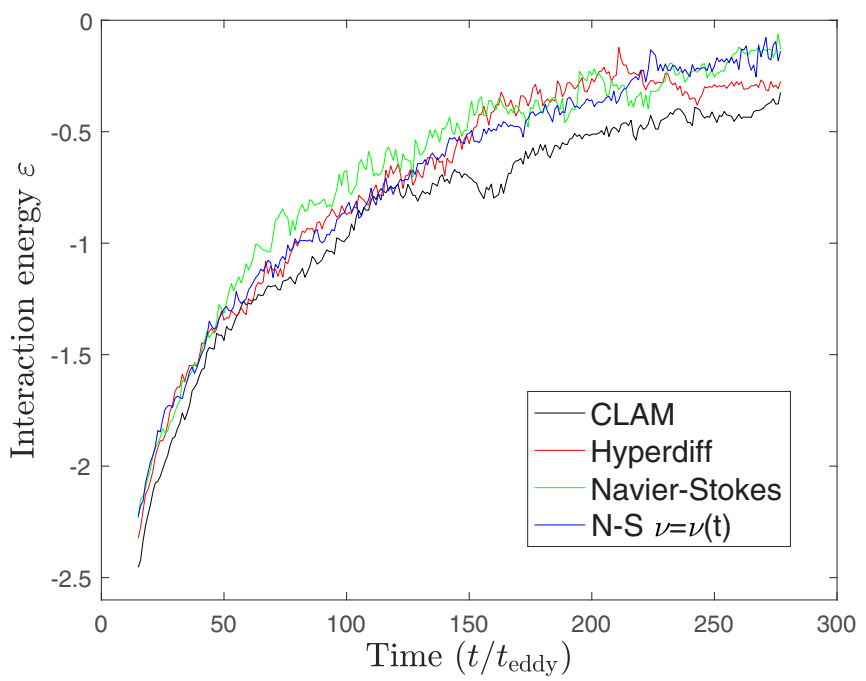

FIG. 2. Ensemble-average normalized vortex interaction energy $\varepsilon(t)$ in the $k_{i}=32$ 2DCT simulations, using different numerical schemes: CLAM (black curve), pseudospectral with hyperdiffusion (red curve), pseudospectral Navier-Stokes with time-varying viscosity (blue curve), and standard pseudospectral NavierStokes (green curve). See text for details. 
independent of the scheme used, except for a tendency for the more dissipative schemes to spin-up with slightly higher $\varepsilon$ at early times, which persists throughout the simulations. It is interesting, and perhaps unexpected, to note that the heating effect is almost unchanged despite significant energy (6.84\% over the main part of the simulation) being lost in the constant $v$ Navier-Stokes simulations.

\section{E. Quasi-equilibrium energy spectrum}

Next, the fact that the GVD model has exactly solvable statistics is exploited (see Appendix 1), following the authors of Refs. [20-22], to calculate an exact $\varepsilon$-dependent quasi-equilibrium energy spectrum. The new method improves upon that found in Ref [2], in which the energy spectrum $\mathcal{E}(k)$ of a vortical flow is calculated by averaging over the uniform ensemble (random vortex positions), resulting in a predicted spectrum $\mathcal{E}_{0}(k)=2 \pi k^{-1} \Pi(k)^{-1}\left(\sim k^{-1}\right.$ at low wave numbers) which is independent of $\varepsilon$. However, it is well-established [5] that 2DCT simulations exhibit energy spectra which can differ significantly from $k^{-1}$ at low wave numbers, and the authors of Ref. [5] proposed a hybrid theory based on incorporating the spectral statistical mechanics found in Refs. [23,24], to account for the discrepancies. The GVD approach taken here dispenses for the need for a such a hybrid theory by introducing $\varepsilon$-dependence into the theory found in Ref. [2].

The spectrum is calculated by first obtaining the density of states $W(\varepsilon)$, which measures the number of GVD microstates with interaction energy $\varepsilon$, as

$$
W(\varepsilon)=\frac{1}{2 \pi} \int_{-\infty}^{\infty} \exp \left[i s\left(\varepsilon+\frac{\mu_{H}}{\sigma_{H}}\right)-\sum_{k}^{\prime} \log \left(1+\frac{\text { is }}{\sigma_{H} k^{2} \Pi(k)}\right)\right] d s
$$

It turns out that the function $W(\varepsilon)$ is relatively insensitive to $\Pi(k)$ in the regime of the simulations (see Fig. 5 below), and remains close to its point vortex limit [22,25]. Next, following Ref. [22], the equilibrium energy in mode $\boldsymbol{k}$ is found to be

$$
\left\langle E_{\boldsymbol{k}}\right\rangle=\frac{1}{k^{2} \Pi(k)}\left(1-\frac{1}{W(\varepsilon)} \int_{0}^{\infty} W^{\prime}(\varepsilon-\xi) e^{-k^{2} \Pi(k) \sigma_{H} \xi} d \xi\right),
$$

where $\langle\cdot\rangle$ denotes averages over microstates with fixed $\varepsilon$ (the microcanonical ensemble). From Eq. (7) it is evident that the corresponding equilibrium energy spectrum $\mathcal{E}(k)=2 \pi k \times(7)$ is equal to $\mathcal{E}_{0}(k)$ (as found using the approach of the authors of Ref. [2]) plus a $\varepsilon$-dependent correction term. This correction term determines how the energy surplus or deficit in the system, measured relative to the randomized spectrum, is distributed across wave numbers. A significant success of this quasi-equilibrium theory is that it gives a quantitative prediction of the distribution of energy in the gravest modes of the system, in particular the energy in the $k=1$ modes (the Onsager-Kraichnan condensate $[8,11])$ at negative temperatures. It follows from Eq. (7) that when $\varepsilon \gg 0$, the energy in all modes with $k>1$ becomes saturated, with energy $\left\langle E_{k}\right\rangle_{s}=k^{-2}[\Pi(k)-\Pi(1)]^{-1}$. All remaining energy in the system is then concentrated in the $k=1$ condensate modes.

There is a further necessary condition for decaying 2DCT to converge to the quasi-equilibrium spectrum. The nonequilibrium timescale $\tau_{\text {ne }}$, on which the quasi-equilibrium spectrum $\mathcal{E}(k)$ evolves in time, must be longer than the equilibrium timescale $\tau_{\mathrm{e}}$ on which the system relaxes to the equilibrium spectrum from random initial conditions (as measured in the GVD model). Since Eq. (7) is relatively insensitive to changes in $\Pi(k)$, it follows that $\tau_{\mathrm{ne}}=\dot{\varepsilon}^{-1}$. The timescale $\tau_{\mathrm{e}}$ can be identified with the timescale on which the energy in the gravest modes (i.e., $E_{k}$ for $k=1$ ) evolves under the dynamics, which is also the timescale for a typical vortex to cross the domain. The time for a single vortex to travel a typical vortex separation distance $r_{v}$ is $\tau_{\mathrm{s}}=r_{v}^{2} \omega_{0}^{-1} a_{m}^{-1}$, where $\omega_{0}$ is a vorticity scale, and $a_{m}$ a (maximum) vortex area scale. Under the assumption that every $\tau_{\mathrm{s}}$ the vortex is scattered and moves in a new random direction, it will take time $\tau_{s} l^{2} r_{v}^{-2}$ to travel an expected distance $l \gg r_{v}$, giving $\tau_{\mathrm{e}} \sim \omega_{0}^{-1} a_{m}^{-1}$ if the domain is to be crossed (i.e., $l=1$ ). 

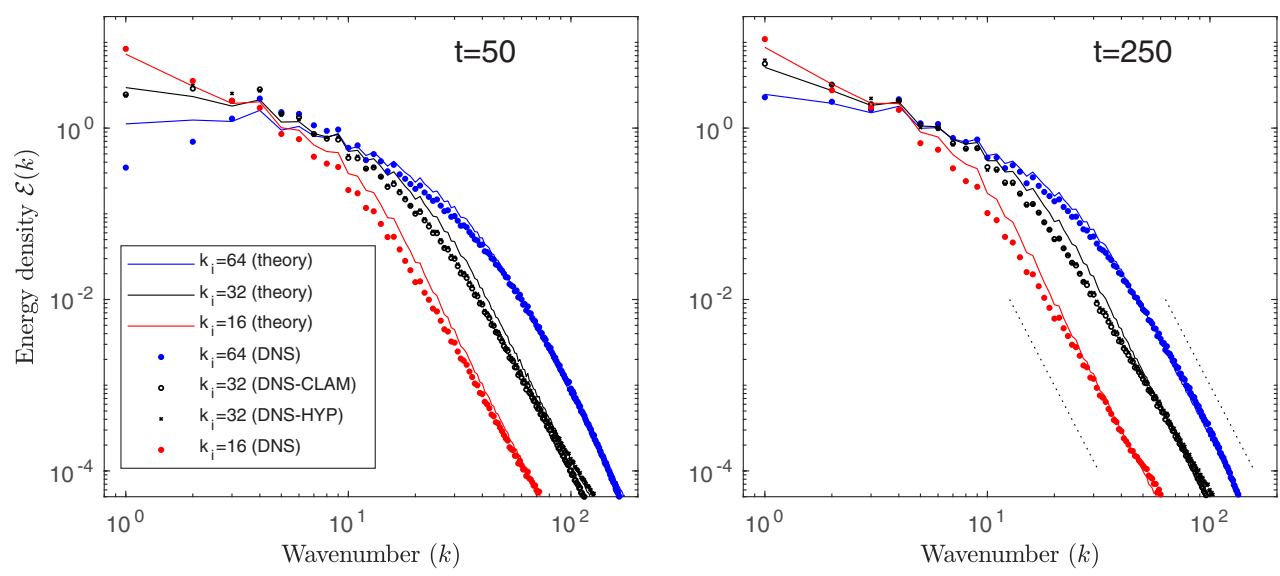

FIG. 3. Ensemble-average energy spectra from the DNS (points) versus predictions from the equilibrium statistical theory (lines) at $t=50 t_{\text {eddy }}$ (left) and $t=250 t_{\text {eddy }}$ (right). The dotted lines show a $k^{-5}$ high-wavenumber spectrum as predicted by DSMGT. For initial data wave number $k_{i}=32$, results from both the CLAM (circles) and hyperdiffusion (crosses) DNS are given.

\section{F. Energy spectrum: Predictions versus DNS}

Figure 3 compares ensemble-averaged energy spectra in the 2DCT DNS (points) with theoretical predictions (curves), at early and late times in the simulations. The theoretical spectra are calculated using the Gaussian vortices identified in the DNS (see above) to find a typical vorticity scale $\omega_{0}(t)$, and to fit to a vortex area distribution [4]

$$
n(a, t)=c(t)\left\{\begin{array}{cc}
a^{-1} & a<a_{m}(t), \\
0 & \text { otherwise }
\end{array}\right.
$$

where $n(a, t)$ is the number density of vortices with area $a$ (see Appendix 3 for details). The function $\Pi(k)$ is then calculated from Eqs. (8), and Eqs. (6) and (7) are then used to obtain $\mathcal{E}(k)$, with $\varepsilon$ taken from Fig. 1. The theory matches the DNS with excellent agreement except where the vortex population is evolving rapidly, as seen in the $k_{i}=64$ ensemble at $t=50 t_{\text {eddy }}$, when the equilibrium assumption $\tau_{\mathrm{ne}} \gg \tau_{\mathrm{e}}$ is not valid.

\section{G. Constraints on vortex evolution laws}

Various (semi)empirical laws, reviewed in Ref. [26], were proposed to describe the time evolution of the vortex population in 2DCT. In particular, using similarity arguments [4] proposed the law (8) with $\omega_{0}$ constant, $c(t) \sim t^{-2 / 3}$ and $a_{m}(t) \sim t^{1 / 3}$ (the DSMGT law hereafter). A key result of this work is that a given law, such as DSMGT, can be explicitly identified with a trajectory for $\varepsilon(t)$ (see Appendix 2). In the case of laws of the type (8), $\varepsilon(t)$ evolves according to Eq. (4) with $H=E$ constant and

$$
\mu_{H}=\pi \omega_{0}^{2} c a_{m}^{2} G\left(k_{0} a_{m}^{1 / 2}\right), \quad \sigma_{H}=\frac{\pi^{1 / 2} \omega_{0}^{2} c a_{m}^{2}}{\sqrt{8} k_{0}}
$$

where to leading order $G(x)=\left(1-2 \gamma-2 \log x^{2}\right) / 8$ and $k_{0} \approx 0.663$ is constant. Equation (9) reveals that, because DSMGT has $c a_{m}^{2}$ constant, it has the special property of being on the boundary between heating and cooling (in fact, $\varepsilon$ has only logarithmic time dependence), consistent with the similarity assumptions underpinning DSMGT. In Fig. 4, however, it is clear the logarithmic DSMGT heating rate is much lower than that observed in the DNS. A more general law with $\omega_{0}$ constant, $a_{m}(t) \sim t^{\mu}$ and $c(t) \sim t^{\nu}$ has leading behavior $\varepsilon \sim t^{-2 \mu-\nu}$. The heating observed in the DNS constrains $-2 \mu-v \approx 0.11$, and a close fit to the DNS data in Fig. 1. which satisfies this is 


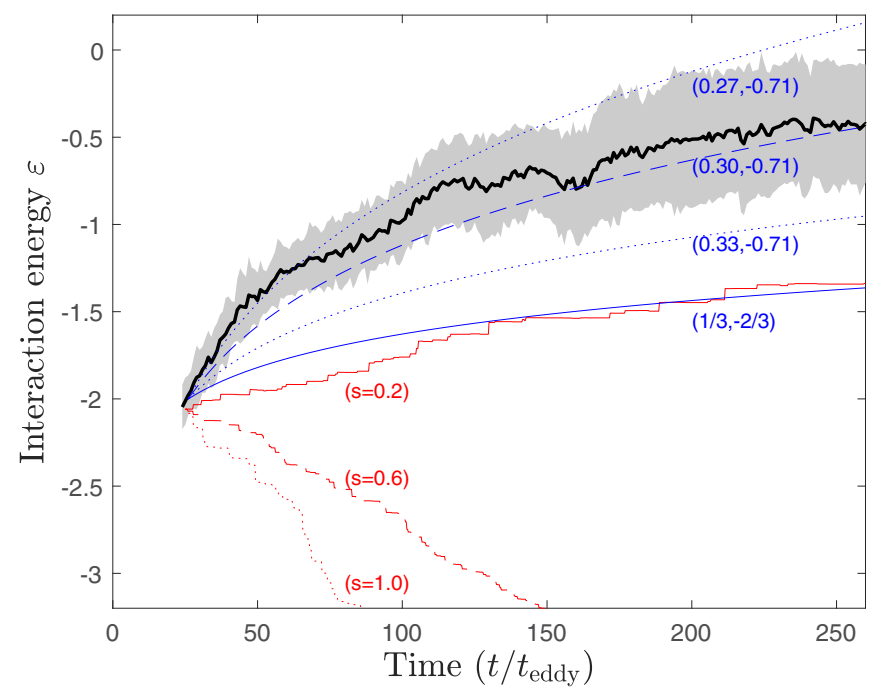

FIG. 4. Time evolution of $\varepsilon$ in the DNS (solid black curve ensemble mean, grey area \pm 1 standard deviation), versus predictions of Eq. (9) for vortex distributions of the form (8) evolving according to temporal power laws [solid blue curve, DSMGT law; dashed blue curve, fit with $(\mu, \nu)=(0.30,-0.71)$; dotted blue curves $(\mu, \nu)=(0.30 \pm 0.03,-0.71)]$. Red curves show results from the fluid energy conserving punctuated GVD model with $q=3$ and $s=0.2$ (solid red) $s=0.6$ (dashed red) and $s=1$ (dotted red).

$(\mu, v)=(0.30,-0.71)$. It is notable that the majority of vortex number decay laws obtained from DNS (see Table I of Ref. [26]) have $v \approx-0.71$. In summary the observed heating constrains the decay rate to be faster than the $v=-2 / 3$ prediction of DSMGT.

\section{H. Constraints on aggregation models}

Another approach [12-14,26,27] to understanding 2DCT is to use a simplified vortex dynamics together with a kinetic aggregation law for vortex merger. Most previous studies used the point vortex framework, which means that the resulting "punctuated equilibrium" models for 2DCT are underconstrained because no account is taken of the conservation of the total fluid energy during merger events. The GVD model, by contrast, can be augmented with a fluid energy-conserving vortex merger process, in which energy conservation is achieved by careful control of the position of the merged vortex, see Appendix 4 for details. A number of integrations of this GVD model have been compared to investigate the effect on $\dot{\varepsilon}$. The results are highly sensitive to the details of the chosen merger law. A two-parameter $(q, s)$ law was investigated in detail: two like-signed vortices with radii $r_{i}$ and circulations $\Gamma_{i}=2 \pi \omega_{0} r_{i}^{2}\left(i=1,2,\left|\Gamma_{1}\right| \geqslant\left|\Gamma_{2}\right|\right)$ are merged when they come within a distance $q\left(r_{1}^{2}+r_{2}^{2}\right)^{1 / 2}$ and the merged vortex is assigned circulation $\Gamma_{1}+s \Gamma_{2}$. That is, the parameter $q \in[2.5,3.5]$ controls the merger distance, and $s \in[0,1]$ the amount of vorticity mixed into the background during mergers [28]. The heating rate turns out to be relatively insensitive to $q$, but as seen in Fig. 4 is strongly sensitive to $s$. Positive heating is found only when the vorticity loss rate in mergers is so high as to be unphysical $(s=0.2)$, and vorticity-conserving mergers ( $s=$ 1) lead to rapid cooling. The results support the "mergers lead to cooling" argument given in the introduction, and in particular show that the long-time $t^{-1}$ vortex decay found in Ref. [14] arises because rapid cooling has driven the system into the dipole regime $(\varepsilon \ll-1)$.

\section{DISCUSSION AND CONCLUSION}

Our main result is the clear evidence from DNS that decaying 2DCT does not remain self-similar in time, but instead undergoes a persistent statistical heating consistent with like-signed vortices 
gradually becoming more clustered. Compared to evaporative heating in 2DQT, the heating is weak, hence the self-similar theory of DSMGT remains reasonably accurate. However, the observed heating provides a strong constraint on any theory of the evolution of the vortex population, and has been shown to be more consistent with a vortex decay law $\sim t^{-0.71}$ (c.f. Ref. [1]) than DSMGT's $t^{-2 / 3}$. Further, it has been shown that "punctuated equilibrium" aggregation models of vortex mergers lead to rapid cooling (dipole formation). Even when such models are modified [29] to conserve fluid energy, and to allow for significant vorticity loss (i.e., mixing into the background), the observed heating rate cannot be reproduced. Instead, it seems likely that an accurate aggregation model must consider a combination of mergers with vorticity loss (producing modest cooling) and straining out of opposite-signed vortices [6] (producing heating analogous to evaporative heating in 2DQT). In this context it is notable that flows with single-signed vortices [30,31] can result in the formation of "vortex crystals" consisting of a small number of vortices in a stationary configuration, suggesting that if only mergers occur then the system does cool to the lowest attainable value of $\varepsilon$. This conclusion resolves the apparent paradox of the introduction, individual mergers do lead to cooling, but an overall positive heating rate can still drive the system towards a negative temperature end state. A detailed investigation of the relative roles of vortex mergers versus straining will follow in future work.

\section{ACKNOWLEDGMENTS}

This work was begun at the Kavli Institute under Grant No. NSF PHY11-25915. Helen Burgess is thanked for many useful and insightful comments.

\section{APPENDIX: MATHEMATICAL AND TECHNICAL DETAILS}

\section{The density of states function (6) and equilibrium energy spectrum (7)}

The GVD density of states function $W(\varepsilon)$, given by Eq. (6), can be identified with be the pdf of the random variable associated with the normalized Hamiltonian

$$
\bar{H}=\frac{H-\mu_{H}}{\sigma_{H}}
$$

under the uniform ensemble. The uniform ensemble is defined to be the ensemble of vorticity distributions $\omega(\boldsymbol{x})=\sum_{i=1}^{N} \omega_{i}\left(\left|\boldsymbol{x}-\boldsymbol{X}_{i}\right|\right)$ generated when the positions of the vortex centroids $\mathbf{X}_{i}$ are taken to be random variables uniformly distributed in $\mathcal{D}$. Notice that by construction $\bar{H}$ has zero mean and unit variance.

To calculate $W(\varepsilon)$ is useful to first consider the Fourier expansion of the vorticity field

$$
\begin{gathered}
\omega(\boldsymbol{x})=\frac{1}{2 \pi} \sum_{\boldsymbol{k}} \Omega_{\boldsymbol{k}} e^{i \boldsymbol{k} \cdot \boldsymbol{x}} \\
\Omega_{\boldsymbol{k}}=\frac{1}{2 \pi} \int_{\mathcal{D}} \omega(\boldsymbol{x}) e^{-i \boldsymbol{k} \cdot \boldsymbol{x}} d \boldsymbol{x}=\sum_{i=1}^{N} \hat{\omega}_{i}(k) e^{-i \boldsymbol{k} \cdot \boldsymbol{X}_{i}}
\end{gathered}
$$

The final equality exploits the fact that the vortices are much smaller than $\mathcal{D}$. The explicit form of the Green's function

$$
G\left(x, x^{\prime}\right)=-\sum_{k} \frac{e^{i k \cdot\left(x-x^{\prime}\right)}}{(2 \pi k)^{2}},
$$

can be used to write $H$ in terms of the $\Omega_{k}$,

$$
H=\sum_{k} \frac{\left|\Omega_{k}\right|^{2}}{k^{2}} .
$$



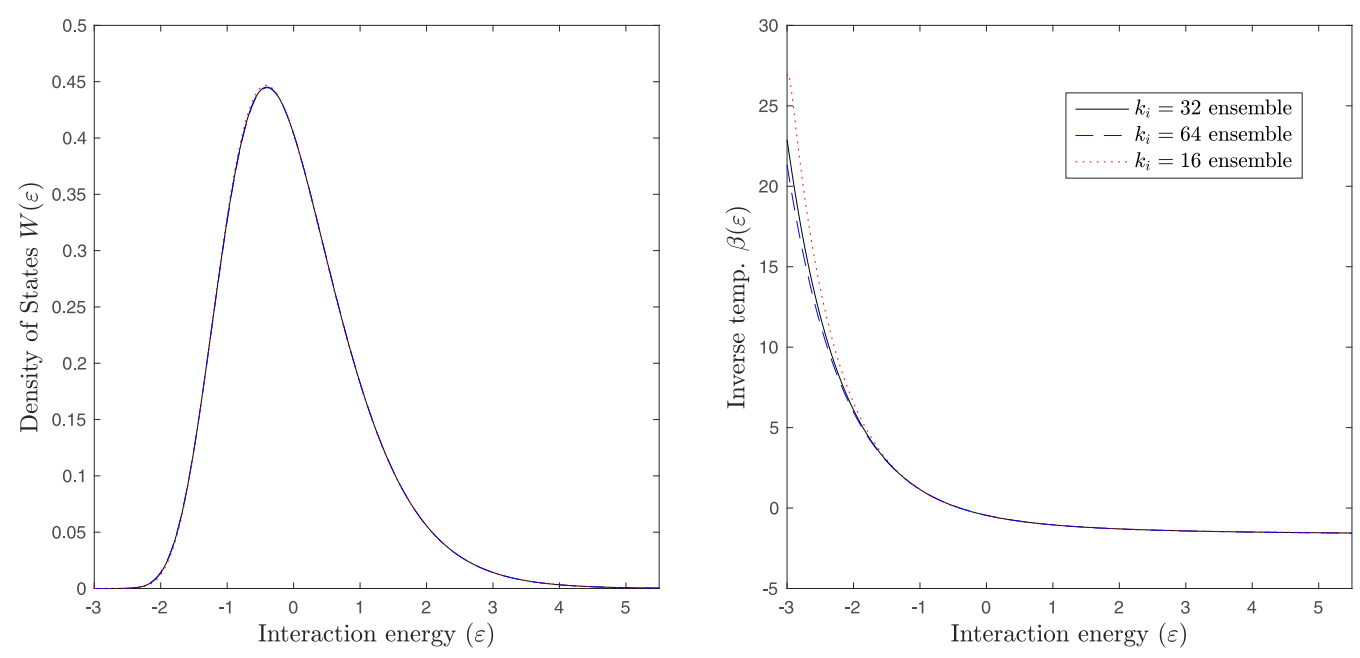

FIG. 5. Left: Density of states function $W(\varepsilon)$ in the GVD system, calculated for vortices with areas distributed according to Eq. (8), with the free parameters $\left(a_{m}, c, \omega_{0}\right)$ chosen as a best fit to the DNS results at $t=50$ for the $k_{i}=32$ ensemble (solid black curve), the $k_{i}=64$ ensemble (dashed blue curve) and the $k_{i}=16$ ensemble (dotted red curve). Right: The corresponding (scaled) inverse temperature $\beta(\varepsilon)=W^{\prime}(\varepsilon) / W(\varepsilon)$.

The expression for $\mu_{H}=\langle H\rangle_{0}$ in Eq. (5) is now obtained by taking the expected value of

$$
\left|\Omega_{\boldsymbol{k}}\right|^{2}=\sum_{i=1}^{N} \sum_{j=1}^{N} \hat{\omega}_{i}(k) \hat{\omega}_{j}(k) \cos \left[\boldsymbol{k} \cdot\left(\boldsymbol{X}_{i}-\boldsymbol{X}_{j}\right)\right]
$$

under the uniform ensemble. The corresponding expression for $\sigma_{H}^{2}$ is found similarly by considering $\left|\Omega_{k}\right|^{4}$.

Treating the $\Omega_{k}$ in Eq. (A3) as a sum of independent random variables, the Lyapunov central limit theorem can be used in the limit $N \rightarrow \infty$ to write

$$
\Omega_{k}=\frac{1}{\Pi(k)^{1 / 2}} \frac{\left(A_{k}+i B_{k}\right)}{2^{1 / 2}} \quad \text { where } \quad A_{k}, B_{k} \sim \mathcal{N}(0,1)
$$

It follows that $\left|\Omega_{k}\right|^{2} \sim \Pi(k)^{-1} C_{k}$, where $C_{k} \sim \exp (1)$ is an exponential random variable with pdf $p(x)=e^{-x}(x>0)$. The scaled Hamiltonian $\bar{H}$ is now a weighted sum of independent exponential random variables, shifted to have zero mean,

$$
\bar{H}=\sum_{k}^{\prime} \frac{C_{k}-1}{\sigma_{H} \Pi(k) k^{2}} .
$$

The pdf of the sum (A8) can be obtained using the standard Fourier transform method, following $[21,22]$, leading to Eq. (6).

Figure 5 shows $W(\varepsilon)$ calculated using Eq. (6) for different vortex distributions typical of the DNS (see caption for details). It turns out that $W(\varepsilon)$ is almost independent of the specific parameters chosen, and remains very close to that of the point vortex system. The corresponding inverse temperatures $\beta=W^{\prime} / W$ (right panel) show the single-valued dependence of $\beta$ on $\varepsilon$. These curves do show some sensitivity to the details of the vortex area distribution, but only at negative interaction energies.

The formula for the equilibrium energy spectrum (7) can then be obtained from an expression of Bayes' theorem [22]. Consider the microcanonical pdf $p_{\varepsilon}\left(e_{k}\right)$ of $E_{k}=\left|\Omega_{k}\right|^{2} / k^{2}$, where the microcanonical ensemble is defined to be all states with $\bar{H}=\varepsilon$. This can be interpreted as a 
conditional pdf under the uniform ensemble and, using Bayes, expressed as

$$
p_{\varepsilon}\left(e_{k}\right)=\frac{W_{-k}\left[\varepsilon+\sigma_{H}^{-1}\left(e_{0 k}-e_{k}\right)\right]}{W(\varepsilon)} k^{2} \Pi(k) e^{-k^{2} \Pi(k) e_{k}} .
$$

Here $W_{-\boldsymbol{k}}(\cdot)$ denotes the density of states function (6) evaluated with wave number $\boldsymbol{k}$ omitted from the calculation. The $W_{-\boldsymbol{k}}$ term in Eq. (A9) is the conditional pdf for $\bar{H}$ given that $E_{\boldsymbol{k}}=e_{k}$. The constant $e_{0 k}=\left\langle E_{\boldsymbol{k}}\right\rangle_{0}=\Pi(k)^{-1} k^{-2}$, and the exponential term in Eq. (A9) is the pdf $p_{0}\left(e_{k}\right)$ of $E_{\boldsymbol{k}}$ under the uniform ensemble.

The equilibrium energy spectrum is obtained by taking the expectation of the pdf (A9),

$$
\left\langle E_{\boldsymbol{k}}\right\rangle=\int_{0}^{\infty} e_{k} p_{\varepsilon}\left(e_{k}\right) d e_{k} .
$$

The resulting expression is simplified to Eq. (7) using the substitution $\xi=e_{k} / \sigma_{H}$ and the convolution identities given in Ref. [22].

The saturation spectrum result follows from taking the limit $\varepsilon \rightarrow \infty$ in Eq. (7). As $\varepsilon \rightarrow \infty$, most energy ends up in the $k=1$ condensate mode, and it follows that

$$
W(\varepsilon) \sim \varepsilon \Pi(1)^{2} e^{-\Pi(1) \varepsilon}, \quad \text { as } \varepsilon \rightarrow \infty .
$$

Inserting Eq. (A11) into Eq. (7) and integrating results in the expression for $\lim _{\varepsilon \rightarrow \infty}\left\langle E_{k}\right\rangle=\left\langle E_{k}\right\rangle_{s}$ given in the main text.

\section{Evolution of $\varepsilon(t)$ under the vortex evolution law (8)}

Here it will be shown explicitly that a vortex evolution law of the form (8) completely determines the time evolution of $\varepsilon(t)$, under the reasonable assumption of conservation of fluid energy. First, the function

$$
\Pi(k)=\left(\sum_{i=1}^{N} \hat{\omega}_{i}(k)^{2}\right)^{-1},
$$

where $\hat{\omega}_{i}(k)$ is the Hankel transform of the axisymmetric vorticity $\omega_{i}(r)$ of the $i$ th vortex, must be determined. For simplicity we assume the vortices to be Gaussian with constant vorticity maxima $\omega_{0}$, although these assumptions are easily relaxed. In this case

$$
\omega_{i}(r)=\omega_{0} \exp \left(-\frac{r^{2}}{2 a_{i}}\right)
$$

and

$$
\hat{\omega}_{i}(k)=\omega_{0} a_{i} \exp \left(-\frac{k^{2} a_{i}}{2}\right)
$$

The function $\Pi(k)$ can now be straightforwardly evaluated by substituting an integral for the sum in its definition, giving

$$
\Pi(k)=\left(\int_{0}^{\infty} \hat{\omega}(k, a)^{2} n(a) \mathrm{d} a\right)^{-1}=\frac{F\left(k a_{m}^{1 / 2}\right)}{c \omega_{0}^{2} a_{m}^{2}},
$$

where

$$
F(x)=\frac{x^{4}}{1-\left(1+x^{2}\right) \exp \left(-x^{2}\right)} .
$$

Next, the evolution of $\mu_{H}$ can be estimated from Eq. (A15) by replacing the primed sum in Eq. (5) by an integral

$$
\mu_{H} \approx \pi \int_{k_{0}}^{\infty} \frac{k d k}{k^{2} \Pi(k)}=\pi c \omega_{0}^{2} a_{m}^{2} G\left(k_{0} a_{m}^{1 / 2}\right),
$$


where

$$
G(x)=\int_{x}^{\infty} \frac{d s}{s F(s)}=\frac{\left(1-2 \gamma-2 \log x^{2}\right)}{8}+O\left(x^{2}\right) .
$$

Choosing $k_{0}=0.663$ gives a good fit to the sum over the range of $a_{m}$ seen in the simulations. Since $a_{m} \approx 0.001-0.01$ in the DNS, and $x=k_{0} a_{m}^{1 / 2}$, the small $x$ limit for the integral is well-justified. Similarly,

$$
\sigma_{H}^{2} \approx \pi \int_{k_{0}}^{\infty} \frac{k d k}{k^{4} \Pi(k)^{2}} \approx \frac{\pi c^{2} \omega_{0}^{4} A_{m}^{4}}{8 k_{0}^{2}}
$$

giving the formulas used in Eq. (9). Predictions for the time-evolution of $\varepsilon$ then follow from power-law assumptions for the evolution of $c$ and $A_{m}$, and the fact that $E$ remains constant in the simulations.

\section{Fitting the DSMGT distribution to the DNS}

Figure 1 shows the evolution of $c(t)$ and $a_{m}(t)$ calculated from the DNS. These quantities are obtained by first fitting a DSMGT distribution of vortices, given by Eq. (8), to the instantaneous DNS output. For the calculations of the theoretical spectra of Fig. 3, the formula (6) is then used to calculate the density of states function $W(\varepsilon)$ used in Eq. (7), using the form of $\Pi(k)$ given in Eq. (A15).

The fit is performed as follows. First, vortices in the DNS are identified using the algorithm of Ref. [4] and are mapped to Gaussian GVD vortices as described in the main text. Only vortices above a minimum area $a_{-}=10^{-3}$ are considered for the calculation. The constant peak vorticity value $\omega_{0}$ is found from the weighted mean (weighted by absolute value of the vortex circulation) of the absolute value of the peak vorticity of these vortices. The number density of vortices is then estimated as $c=N\left[a \in\left(a_{-}, 10 a_{-}\right)\right] / \ln (10)$, where $N[\cdot]$ denotes the count of vortices satisfying a given criterion. Finally the maximum vortex area $a_{m}$ is chosen so that $\mu_{H}$ calculated from Eq. (9) is equal to the value for the exact distribution calculated from Eq. (5). The result for $a_{m}$ in fact corresponds very closely in practice to the area of the largest vortex in the DNS, at least when $N$ is large.

\section{Fluid-energy conserving aggregation law for vortex merger in the GVD model}

The punctuated GVD model solves (2) for $N$ Gaussian vortices with radii $r_{i}$ and circulations $2 \pi \omega_{0} r_{i}^{2}\left(\omega_{0}\right.$ constant). The model runs for discrete time-periods between critical times when the vortex merger criterion, that the separation $r_{i j}$ between the $i$ th and $j$ th vortices is less than the critical distance $q\left(r_{1}^{2}+r_{2}^{2}\right)^{1 / 2}$, is first satisfied for two like-signed vortices. At these critical times, the two merging vortices are instantaneously replaced by a single Gaussian vortex with circulation $\Gamma_{i}+s \Gamma_{j}$ (for $\left|\Gamma_{i}\right|>\left|\Gamma_{j}\right|$, or $s \Gamma_{i}+\Gamma_{j}$ otherwise). The new vortex is first initialized at $\boldsymbol{x}_{n}=\gamma \boldsymbol{x}_{i}+(1-\gamma) \boldsymbol{x}_{j}$, where $\gamma=\left|\Gamma_{i}\right| /\left(\left|\Gamma_{i}\right|+\left|\Gamma_{j}\right|\right)$. However, before the GVD code is restarted, a sequence of small randomly directed perturbations are made to the positions of the new vortex and its neighbors, of amplitude $\Delta=10^{-3}$ for the new vortex, and $\Delta\left(r_{n j} / r_{n}\right)^{2}$ for its neighbors. These random perturbations represent the (otherwise neglected) net effect of the dipolar flow induced by the merging vortices. Working on the assumption that the dipolar flow acts to conserve energy, successive perturbations are accepted if energy of the new configuration is closer to its premerger value, and rejected otherwise. Perturbations are applied until a configuration with the premerger energy is found (by interpolating the final perturbation step as required), and the outcome is a punctuated equilibrium model which conserves fluid energy. 
[1] J. C. McWilliams, The emergence of isolated coherent vortices in turbulent flow, J. Fluid Mech. 146, 21 (1984).

[2] R. Benzi, M. Colella, M. Briscolini, and P. Santangelo, A simple point vortex model for two-dimensional decaying turbulence, Phys. Fluids 4, 1036 (1992).

[3] J. B. Weiss and J. C. McWilliams, Temporal scaling behavior of decaying two-dimensional turbulence, Phys. Fluids A 5, 608 (1993).

[4] D. G. Dritschel, R. K. Scott, C. Macaskill, G. A. Gottwald, and C. V. Tran, Unifying Scaling Theory for Vortex Dynamics in Two-Dimensional Turbulence, Phys. Rev. Lett. 101, 094501 (2008).

[5] D. G. Dritschel, R. K. Scott, C. Macaskill, G. A. Gottwald, and C. V. Tran, Evolution of unforced inviscid two-dimensional turbulence, J. Fluid Mech. 640, 215 (2009).

[6] Z. Xiao, M. Wan, S. Chen, and G. L. Eyink, Physical mechanism of the inverse energy cascade of twodimensional turbulence: a numerical investigation, J. Fluid Mech. 619, 1 (2009).

[7] L. Onsager, Statistical hydrodynamics, Il Nuovo Cimento 6, 279 (1949).

[8] T. P. Billam, M. T. Reeves, B. P. Anderson, and A. S. Bradley, Onsager-Kraichnan Condensation in Decaying Two-Dimensional Quantum Turbulence, Phys. Rev. Lett. 112, 145301 (2014).

[9] The authors of Ref. [8] defined $\varepsilon$ for the point vortex system. Here we extend the definition to a finite core vortex model, allowing $\varepsilon$ to be estimated in 2DCT simulations.

[10] Here $T=W(\varepsilon) / W^{\prime}(\varepsilon)$, see Eq. (6).

[11] T. Simula, M. J. Davis, and K. Helmerson, Emergence of Order from Turbulence in an Isolated Planar Superfluid, Phys. Rev. Lett. 113, 165302 (2014).

[12] G. F. Carnevale, J. C. McWilliams, Y. Pomeau, J. B. Weiss, and W. R. Young, Evolution of Vortex Statistics in Two-Dimensional Turbulence, Phys. Rev. Lett. 66, 2735 (1991).

[13] B. N. Kuvshinov and T. J. Schep, Point-vortex approach in two-dimensional turbulence, Plasma Phys. Rep. 42, 523 (2016).

[14] C. Sire and P.-H. Chavanis, Numerical renormalization group of vortex aggregation in 2D decaying turbulence : the role of three-body interactions, Phys. Rev. E 61, 6644 (2000).

[15] D. Montgomery, W. H. Matthaeus, W. T. Stribling, D. Martinez, and S. Oughton, Relaxation in two dimensions and the "sinh-poisson" equation, Phys. Fluids A 4, 3 (1992).

[16] Z. Yin, D. C. Montgomery, and H. J. H. Clercx, Alternative statistical-mechanical descriptions of decaying two-dimensional turbulence in terms of "patches" and "points", Phys. Fluids 15, 1937 (2003).

[17] D. G. Dritschel and J. Fontane, The combined Lagrangian advection method, J. Comp. Phys. 229, 5408 (2010).

[18] To be precise, point vortex dynamics is recovered by fixing the $\omega_{i}$ to be Dirac delta functions, and omitting the singular $i=j$ terms in the sum for $H$.

[19] Defined as the integral of the square of its vorticity, in the absence of other vortices.

[20] J. G. Esler, T. L. Ashbee, and N. R. McDonald, Statistical mechanics of a neutral point-vortex gas at low energy, Phys. Rev. E 88, 012109 (2013).

[21] J. G. Esler and T. L. Ashbee, Universal statistics of point vortex turbulence, J. Fluid Mech. 779, 275 (2015).

[22] J. G. Esler, The equilibrium energy spectrum of point vortex motion with remarks on ensemble choice and ergodicity, Phys. Rev. Fluids 2, 014703 (2017).

[23] R. H. Kraichnan, Inertial ranges in two-dimensional turbulence, Phys. Fluids 10, 1417 (1967).

[24] D. G. Fox and S. A. Orszag, Inviscid dynamics of two-dimensional turbulence, Phys. Fluids 16, 169 (1973).

[25] Y. B. Pointin and T. S. Lundgren, Statistical mechanics of two-dimensional vortices in a bounded container, Phys. Fluids 19, 1459 (1976).

[26] J. H. LaCasce, The vortex merger rate in freely decaying, two-dimensional turbulence, Phys. Fluids 20, 085102 (2008).

[27] G. Riccardi, R. Piva, and R. Benzi, A physical model for merging in two-dimensional decaying turbulence, Phys. Fluids 7, 3091 (1995).

[28] Here $s=1$ corresponds to no mixing of vorticity into the background, whereas $s=0$ implies that the vorticity in the smaller vortex is completely mixed into the background. 
[29] Note that we did not investigate more sophisticated "three-body" merger laws, such as proposed by, e.g., Ref. [27], however, it seems unlikely to us that these could lead to heating.

[30] K. S. Fine, A. C. Cass, W. G. Flynn, and C. F. Driscoll, Relaxation of 2D Turbulence to Vortex Crystals, Phys. Rev. Lett. 75, 3277 (1995).

[31] D. A. Schecter, D. H. E. Dubin, K. S. Fine, and C. F. Driscoll, Vortex crystals from 2D Euler flow: Experiment and simulation, Phys. Fluids 11, 905 (1999). 